\title{
Implementation of a new 'community' laboratory CD4 service in a rural health district in South Africa extends laboratory services and substantially improves local reporting turnaround time
}

\author{
L M Coetzee, ${ }^{1,2} \mathrm{PhD}$; N Cassim, ${ }^{1,2}$ BTech, MPH; D K Glencross, ${ }^{1,2}$ MB BCh, MMed \\ ${ }^{1}$ National Health Laboratory Service National Priority Programmes, Johannesburg, South Africa \\ ${ }^{2}$ Department of Molecular Medicine and Haematology, Faculty of Health Sciences, University of the Witwatersrand, Johannesburg, South Africa
}

Corresponding author: D K Glencross (debbie.glencross@nhls.ac.za)

Background. The CD4 integrated service delivery model (ITSDM) provides for reasonable access to pathology services across South Africa (SA) by offering three new service tiers that extend services into remote, under-serviced areas. ITSDM identified Pixley ka Seme as such an under-serviced district.

Objective. To address the poor service delivery in this area, a new ITSDM community (tier 3) laboratory was established in De Aar, SA. Laboratory performance and turnaround time (TAT) were monitored post implementation to assess the impact on local service delivery. Methods. Using the National Health Laboratory Service Corporate Data Warehouse, CD4 data were extracted for the period April 2012 July 2013 ( $n=11$ 964). Total mean TAT (in hours) was calculated and pre-analytical and analytical components assessed. Ongoing testing volumes, as well as external quality assessment performance across ten trials, were used to indicate post-implementation success. Data were analysed using Stata 12 .

Results. Prior to the implementation of CD4 testing at De Aar, the total mean TAT was 20.5 hours. This fell to 8.2 hours post implementation, predominantly as a result of a lower pre-analytical mean TAT reducing from a mean of 18.9 to 1.8 hours. The analytical testing TAT remained unchanged after implementation and monthly test volumes increased by up to $20 \%$. External quality assessment indicated adequate performance. Although subjective, questionnaires sent to facilities reported improved service delivery.

Conclusion. Establishing CD4 testing in a remote community laboratory substantially reduces overall TAT. Additional community CD4 laboratories should be established in under-serviced areas, especially where laboratory infrastructure is already in place.

S Afr Med J 2016;106(1):82-87. DOI:10.7196/SAMJ.2016.v106i1.10081

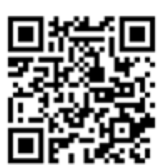

Between April 2014 and March 2015, the South African National Health Laboratory Service (NHLS) CD4 network provided 3.9 million CD4 test results to referring health centres and clinics across South Africa (SA). Although there is an extensive network of $\sim 260$ national laboratories that provide general pathology services and 59 specialised labs that provide CD4 testing, gaps in service have been identified in remote districts (i.e. 14/53 districts) ${ }^{[1]}$ An integrated tiered service delivery model (ITSDM) ${ }^{[1]}$ consisting of six service tiers has been described in line with World Health Organization (WHO) recommendations, ${ }^{[2]}$ which incorporates existing services and provides for extension of services into remote, under-serviced areas to address gaps in service delivery.

ITSDM tiers 4 and 5, at the higher end, are the backbone of existing CD4 services that currently provide high-volume testing in metropolitan areas across SA; a harmonising sixth tier (tier 6) provides for external quality assessment, as well as network and training support. Three new tiers of service are proposed in the ITSDM, to supplement tiers 4 and 5 and enable extended service delivery across the country into areas with service deficiencies. Firstly, tier 3/community laboratories can be established in an existing small general pathology laboratory, processing up to 150 samples per day using traditional or operatorindependent flow cytometry-based technologies (Beckman Coulter XL, ${ }^{[3]}$ Beckman Coulter Aquios ${ }^{[4]}$ and Becton Dickinson FACSCount $\left.(B D S, U S A)^{[5]}\right)$. Secondly, services are further extended with two pointof-care (POC) tiers into areas without reasonable access to a laboratory.
The first POC tier/tier 2 is essentially a POC 'hub' or mini-laboratory that can provide services for up to ten referring community health/ primary health clinics $(\mathrm{CHCs} / \mathrm{PHCs})$ offering antiretroviral therapy within a radius of $50 \mathrm{~km}$ and be managed by a single technician (or recently a Health Professions Council of South Africa-approved cadre 'phlebotomy technician') ${ }^{[6]}$ who will operate multiple POC technologies to provide a range of basic HIV and/or TB services. These facilities can also be implemented into existing small NHLS laboratories and will process $<50$ but $>10$ samples per day, using CD 4 technologies designed to be used at the POC, including FACSPresto (BDS, USA $)^{[]]}$and Pima (Alere, SA). A mini-laboratory can also be set up in a designated area within an existing $\mathrm{CHC}$. All are managed and overseen by the NHLS. The last ITSDM tier 1 provides similar services to tier 2 but will operate in remote, hard-to-reach sites as stand-alone facilities, providing services for the local clinic only. Attending nursing personnel or a phlebotomy technician could take responsibility for the CD4 testing. These extended tiers, which supplement the highvolume testing sites to provide full national service coverage, offer an optimised public health approach to balance overall programmatic costs ${ }^{[8]}$ while lowering and ensuring reliable turnaround time (TAT), irrespective of where the test request originates and in line with local HIV treatment algorithm requirements..$^{[9]}$

Pixley ka Seme, a remote district of SA and one of nine pilot sites for the introduction of National Health Insurance in SA, was identified through ITSDM planning as a district with poor access to CD4 testing. The existing De Aar laboratory in the district 
provides some basic pathology services across a service precinct of $103410 \mathrm{~km}^{2}$ and eight municipalities. The local population of $\sim 186000$ people attend multiple health facilities within a radius of up to $260 \mathrm{~km}$, including ten local hospitals (hospital wards) and $34 \mathrm{PHC}$ clinics. More than $70 \%$ of the population is under the age of 40 years, and the district has an HIV prevalence of $18.4 \%$ (2012 statistics). ${ }^{[10]}$

A tier 3/community laboratory CD4 service was piloted at the existing De Aar NHLS laboratory to address the historically poor CD4 TAT and gaps in service. The impact of the newly implemented tier 3/community CD4 laboratory service in De Aar was assessed by reviewing the TAT before and after the implementation. Success of the implementation was measured through review of ongoing monthly CD4 test volumes and external quality assessment performance of the De Aar laboratory.

\section{Methods}

CD4 testing was implemented and training given at the De Aar laboratory in December 2012 using the Beckman Coulter Epics XL ${ }^{\mathrm{TM}}$ flow cytometry platform and the PanLeucogating method. ${ }^{[3]}$ Using Corporate Data Warehouse (CDW), CD4 sample level data were extracted for the period April 2012 - July 2013 ( $N=11$ 964) for the wider Pixley ka Seme health area. This included pre-implementation data of CD4 workload referred to regional CD4 testing laboratories for testing (Kimberly or Pelonomi) as well as data from the De Aar laboratory itself post implementation (sample volume data were extended to December 2014). The specimen data extract included the laboratory information system (LIS) episode number (which provided evidence of volumes of tests), province, health district and the health facility location name and code from which the sample was sent. Data that were collected with a listed LIS 'tested date' between 1 April and 30 November 2012 were categorised as 'preimplementation'. Data collected after January 2013 were categorised as 'post-implementation'. The reporting measures included CD4 monthly test volumes and mean TATs. To establish TAT, the following LIS date and time fields were used, including: (i) 'registered date, when samples are registered (order entry) at the referring hub or laboratory; (ii) 'test registered date' describing when samples are registered/received at the CD4 testing laboratory; (iii) 'tested date', when the CD4 Epics XLTM CD4 result data are downloaded into the LIS; and lastly, (iv) 'reviewed date', when a medical technologist authorises the CD4 results for printing (by SMS (text message)) at the original referring site. Using the LIS date and time fields, four distinct TAT measures were identified for this analysis: (i) 'PRE-LAB TAT' (pre-analytical component), test registered date minus registered date (interlaboratory or processing hub referral time); (ii) 'IN-LAB TAT', tested date minus test registered date (analytical processing

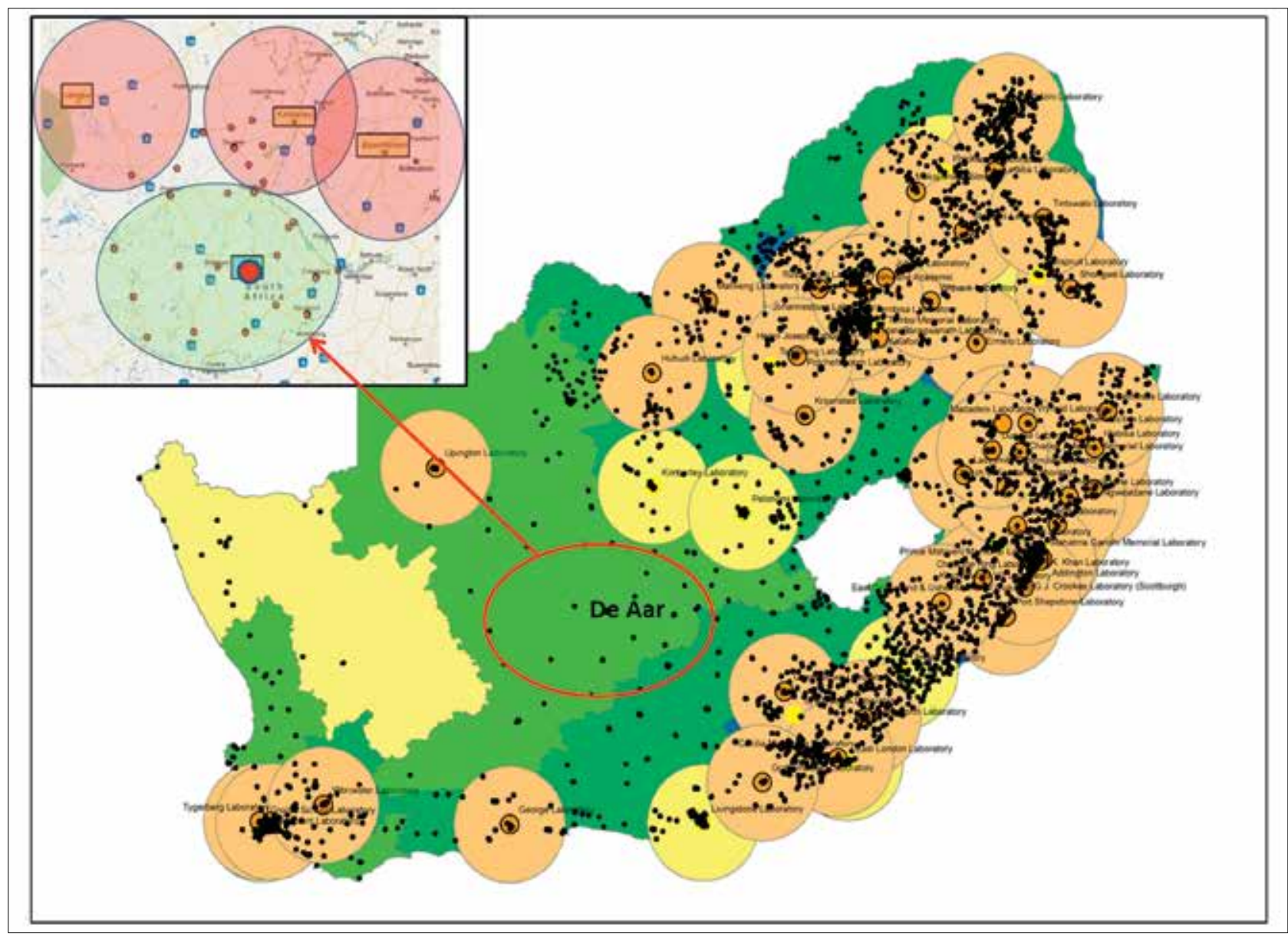

Fig. 1. Map of distribution of existing NHLS CD4 service (as pale yellow and pale orange circular service precincts) in relation to $>4000$ referring PHC clinics (black dots), distributed across 53 defined districts in SA. A new community CD4 service (tier 3) was established in Pixley ka Seme (demarcated as a red circle), at the De Aar NHLS service laboratory in 2012, to address poor access to CD4 testing in this district identified through ITSDM planning. Insert: Map of the greater Pixley ka Seme district reveals existing referring health facilities serviced by the De Aar laboratory. Although the De Aar laboratory offered basic pathology testing, CD4 testing was referred to nearby centralised facilities including Kimberley (243 km away), Upington (433 km) and Bloemfontein/ Pelonomi $(362 \mathrm{~km})$ laboratories. Red circles are estimated existing precinct service coverage. 
time); (iii) 'RVW-TAT', reviewed date minus tested date (time to authorised results following analysis); and lastly (iv) 'TOTAL-TAT', reviewed date minus registered date. Anomalies, due to a computer internal data and time setting for the decentralised Disa ${ }^{\star}$ Lab LIS used previously, that were noted in the LIS date and time fields were excluded (e.g. a reviewed date in the year 1800 (1800/01/01 00:01). This affected only $0.3 \%$ (36/11 964) of the total data set. All data were analysed using Microsoft Excel and Stata 12.

External quality assessment (EQA) performance of the De Aar laboratory on the NHLS CD4 Proficiency Testing (PT)/African Regional External Quality Assessment (AFREQAS) programme ${ }^{[11]}$ was obtained from the De Aar laboratory from trial 19 (May 2013) through to trial 30. Each AFREQAS trial consists of two proficiency material samples, i.e. one normal (CD4 count $>500$ cells $/ \mu \mathrm{L})$ and one low (CD4 count $<200$ cells $/ \mu \mathrm{L}$ ) sample. Performance of the laboratory is measured against the consensus pooled mean result of each trial, and is regarded as adequate if the CD4 result reported by the laboratory falls within 2 standard deviations (SDs) of the consensus pooled trimmed-mean $\mathrm{CD} 4$ result.

\section{Results}

The geographical relationship of 44 referring health facilities and the De Aar laboratory in the Pixley ka Seme district to the preimplementation referral CD4 labs in the Free State (Pelonomi) or Kimberly (North West) is shown in Fig. 1. This figure also gives information about existing CD4 service coverage in SA, showing paucity of local services in the Pixley ka Seme district, in contrast to the relatively well-serviced areas elsewhere in the country. Fig. 2 reveals the impact on TAT of longer (shorter) distances required to refer samples for testing elsewhere; the monthly mean TOTAL-TAT for Pixley ka Seme before implementation ranged from 12 to 38 hours (the majority of samples were referred from the De Aar laboratory to the Kimberley laboratory for testing). A marked difference in mean TOTAL-TAT was noted, reducing from a median of 20.45 hours before implementation to 8.17 hours after December 2012 (Fig. 2). Following a 2-month settling-in period, by March 2013 (mean TOTAL-TAT of 16 hours was recorded), the TOTAL-TAT steadily decreased to a mean of 12 hours and stabilised at 5 hours or less by May 2013. This trend has continued beyond the TAT data extraction period (data not shown).

To assess what component of TAT contributed to TOTAL-TAT, the various components of TAT were assessed including PRE-LAB (pre-analytical), IN-LAB and RVW-TAT (both analytical), before (pre-) and after (post-) implementation (Fig. 2). The pre-analytical component of the TOTAL-TAT was noted to contribute the largest proportion of TOTAL-TAT in the pre-implementation time period. During these pre-implementation months, April - November 2012, pre-analytical TAT showed marked daily variability (with a range of TAT from 4 hours in April 2012 extending to 45 hours in October 2012). After the De Aar CD4 lab was established, the mean PRE-LAB decreased from an average of 18.99 hours to 1.82 hours, reducing the mean monthly TOTAL-TAT substantially. By June 2013, the mean pre-analytical TAT had stabilised at $\sim 2$ hours. Evidence of an initial learning curve was revealed during the first 2 months, i.e. January

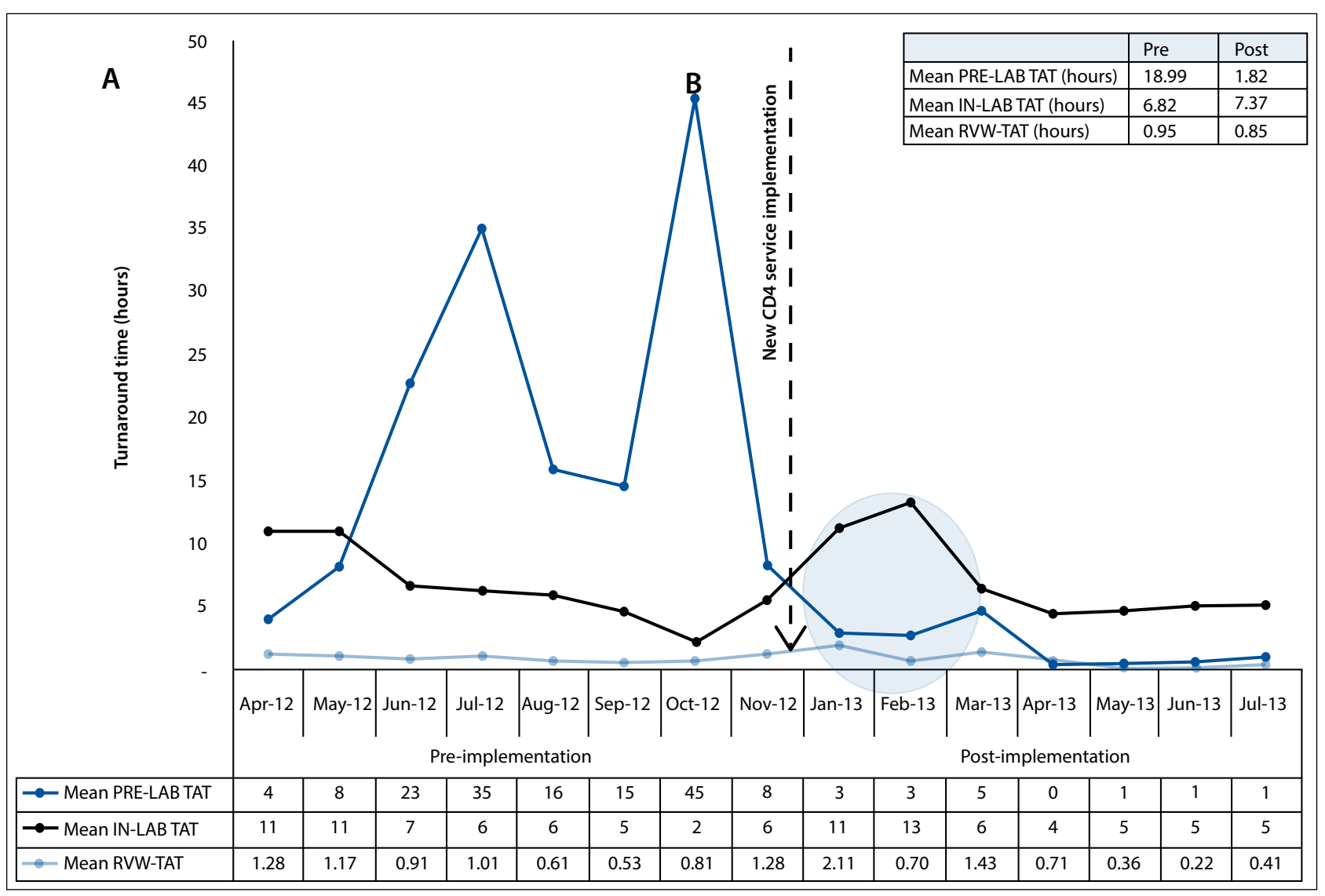

Fig. 2. Breakdown of TOTAL-TAT in the pre-implementation (April - November 2012) and post-implementation phases (January - July 2013), into mean PRE-LAB TAT (blue), mean IN-LAB TAT (black) and mean RVW-TAT (grey). In the pre-implementation phase, the mean PRE-LAB TAT was 4 - 45 hours and the mean IN-LAB TAT 2 - 11 hours. In contrast, in the post-implementation period, the mean PRE-LAB TAT decreased to $<6$ hours but with no significant change in mean IN-LAB TAT at 4 - 13 hours (expected because of use of standardised testing protocols). 
and February 2013, where an IN-LAB TAT of 11 and 13 hours was noted respectively (highlighted in Fig. 2). However, the IN-LAB/ analytical TAT subsequently decreased to that expected of a typical CD4 laboratory processing time as staff became more familiar and integrated the new CD4 testing into their existing workflow (attributable to the adherence to the standardised NHLS CD4 testing procedures that are used irrespective of where testing is performed across the NHLS CD4 network). Further analysis of the TOTAL-TAT data revealed that before implementation of the De Aar CD4 site, 54\% of CD4 samples were reported within a TOTAL-TAT of $\leq 12$ hours. After implementation however, $81 \%$ of samples had a TOTAL-TAT of $\leq 12$ hours (Fig. 2). In a detailed subanalysis, CD4 sample results with a TOTAL-TAT between 13 and 24 hours decreased from $26 \%$ to $13 \%$; those with a TOTAL-TAT $>24$ hours, decreased by $150 \%$, from $19 \%$ to $6 \%$ of all samples tested. Overall, the percentage of samples with a $\leq 24$ hours TOTAL-TAT increased from $81 \%$ before implementation to $94 \%$ post implementation.

Details of month-to-month variation of workload volume post implementation can be seen in Fig. 3, extending to December 2014. Pre-implementation volumes were divided into 'referred to and tested at Kimberley' (mean of 571 samples/month) and 'referred by De Aar and tested in Kimberley' (mean 112 samples per month). After CD4 testing started at De Aar in December 2012, some samples were still referred through De Aar to be tested in Kimberley ( 125 per month). This was attributed to periods of downtime on the instrument (owing to long distances, reaction time of engineers to get instruments operational is prolonged) and/or availability of staff to operate the system. However, the majority of samples from the district are now tested at the De Aar laboratory (monthly mean of 358 samples). Some samples are still referred directly to Kimberley at a mean rate of 287 per month and reflect NHLS business decisions to optimally refer testing from sites that lie far north in Pixley ka Seme to the nearest testing facility (in this instance Kimberley).

\section{External quality assessment performance}

During the post-implementation period of this study, ten trials (20 EQA samples) were tested by the De Aar laboratory. Across each trial, proficiency panel results (CD4 normal and CD4 low, as CD4 count and $\mathrm{CD} 4 \%$ of lymphocytes $(\mathrm{CD} 4 \% \mathrm{~L}))$ were recorded within the required 2 SDI range (Fig. 4 , shaded area; $-2-+2$, with zero as the ideal target). There was an initial outlier EQA result on the normal $\mathrm{CD} 4 \% \mathrm{~L}$ (intervention revealed a transcription error due to inexperience of staff filling in the EQAS submission form). The average SDI of the De Aar CD4 laboratory across all trials was -0.21 , confirming that performance was well within the expected range. However, SDI results from Trial 23 fell outside the expected range for both absolute count and $\mathrm{CD} 4 \% \mathrm{~L}$ (intervention and corrective action revealed that new staff had been appointed without proper EQAS training). The laboratory has sustained acceptable performance on the scheme for the remaining reported trials (Fig. 4).

\section{Discussion}

CD4 testing has been used for establishing immune suppression and disease progression in HIV-positive patients, enabling identification of those patients who are eligible for antiretroviral therapy and/ or treatment for opportunistic infection. Current SA treatment guidelines $^{[9]}$ require that newly identified HIV-positive patients return to the clinic facility within 7 days of testing HIV-positive ${ }^{[12]}$ to

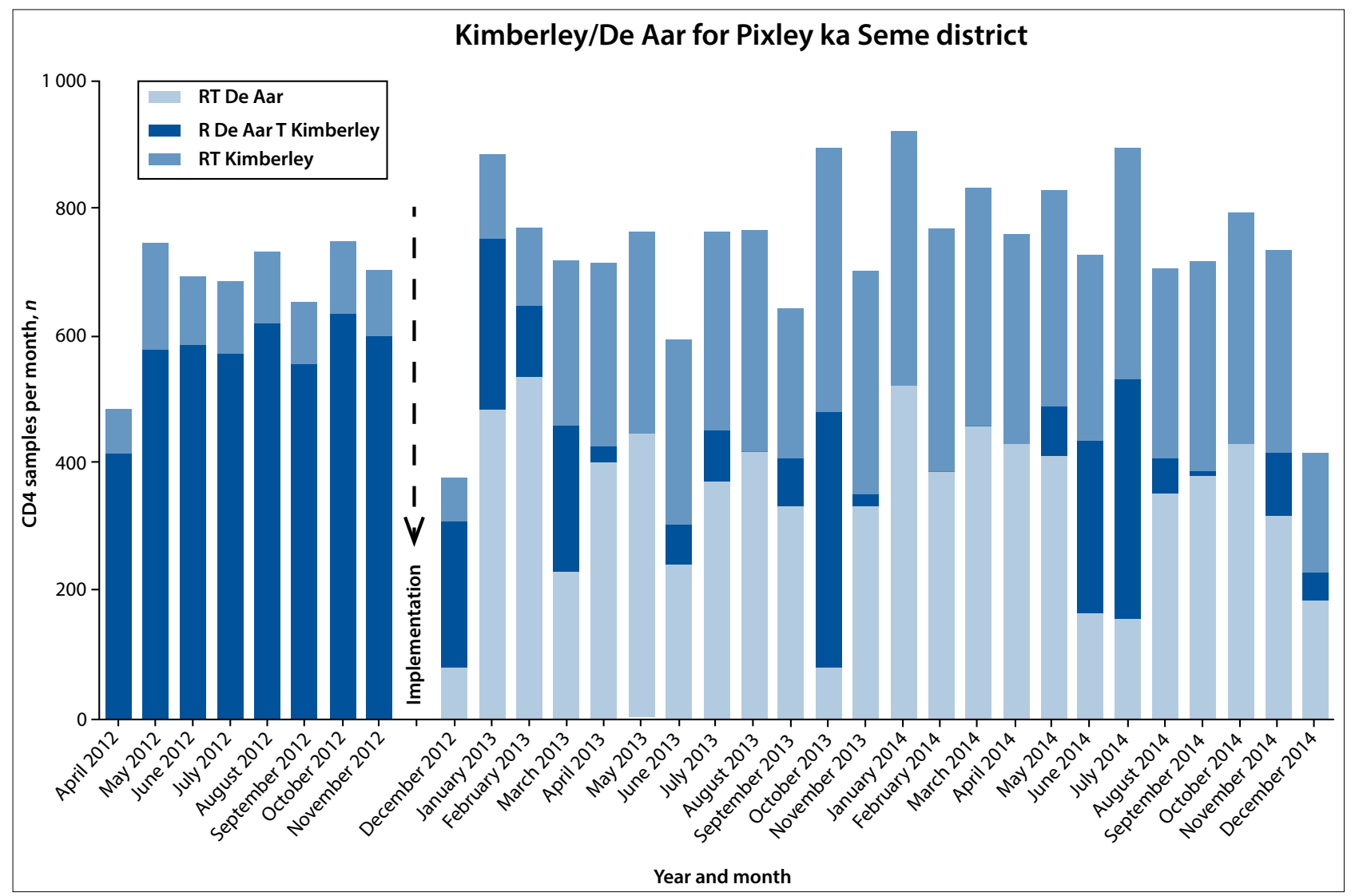

Fig. 3. Total volumes of CD4 tests performed per month before (pre-) and after (post-) CD4 laboratory implementation at De Aar. Volumes of samples 'referred to and tested at Kimberley' ('RT Kimberley'), are shown in contrast to samples 'referred to and tested at De Aar' ('RT De Aar'). A small fraction of samples referred to De Aar for testing is still tested at Kimberley ('R De Aar T Kimberley'/'RT Kimberley'). 
receive the results of the required pathology work-up, including CD4 results outlined in the treatment guidelines. Since the commencement of the South African HIV/ AIDS Comprehensive Care, Management and Treatment programme, laboratorybased CD4 testing has been the mainstay of the CD4 services provided. ${ }^{[3,13]}$ Locally, the NHLS provides access to the majority of these CD4 results within a 24-hour TAT to facilitate algorithm management. ${ }^{[1]}$ In the rural areas of the Northern Cape Province, the closest tier 4/regional and tier 5/metro centralised laboratories are too far (up to $300-400 \mathrm{~km}$ ) away to ensure reliable CD4 TATs. ${ }^{[1]}$

Several service delivery options were considered for Pixley ka Seme area that would improve TAT, while taking into account the vast distances between facilities in the district and proximity to the closest testing facilities. The first service option considered CD4 testing on a mobile unit, ${ }^{[14]}$ with various scenarios proposed. These included a once-per-month service option (costing ZAR125.47/test), a fortnightly option (ZAR201.22/test/visit), a weekly option (ZAR352.73) as well as a daily service delivery option (ZAR1 564.75/test). ${ }^{[14]}$ This approach was abandoned because of the prohibitively high, unaffordable cost per test required to maintain reasonable access to services on at least 4 days a week.

Implementation of widespread POC CD4 testing services in all 44 referring facilities was also considered as the second option, potentially providing immediate patient access to results and purporting the added advantage ${ }^{[13]}$ of improving the number of patients enrolled into care. Aside from the logistical and other problems of widespread POC technology implementation, ${ }^{[1]}$ overall voluntary counselling and testing process compliance has been reportedly poor, ${ }^{[15]}$ with specific areas of concern including specimen collection methodology and availability of equipment, e.g. gloves, etc., to perform the test (procurement challenges), poor or misuse of available consumables, poor adherence to stipulated incubation times before reading HIV rapid test results, lack of staff training and inconsistent or absent quality assurance practices. ${ }^{[15]}$ This approach was also abandoned in the NHLS because of the substantively higher programmatic costs that could be incurred by implementing POC across multiple clinics; a CD4 test performed at the POC was shown to cost 5 - 7 times more than providing a CD4 test in a laboratory. ${ }^{[8]}$

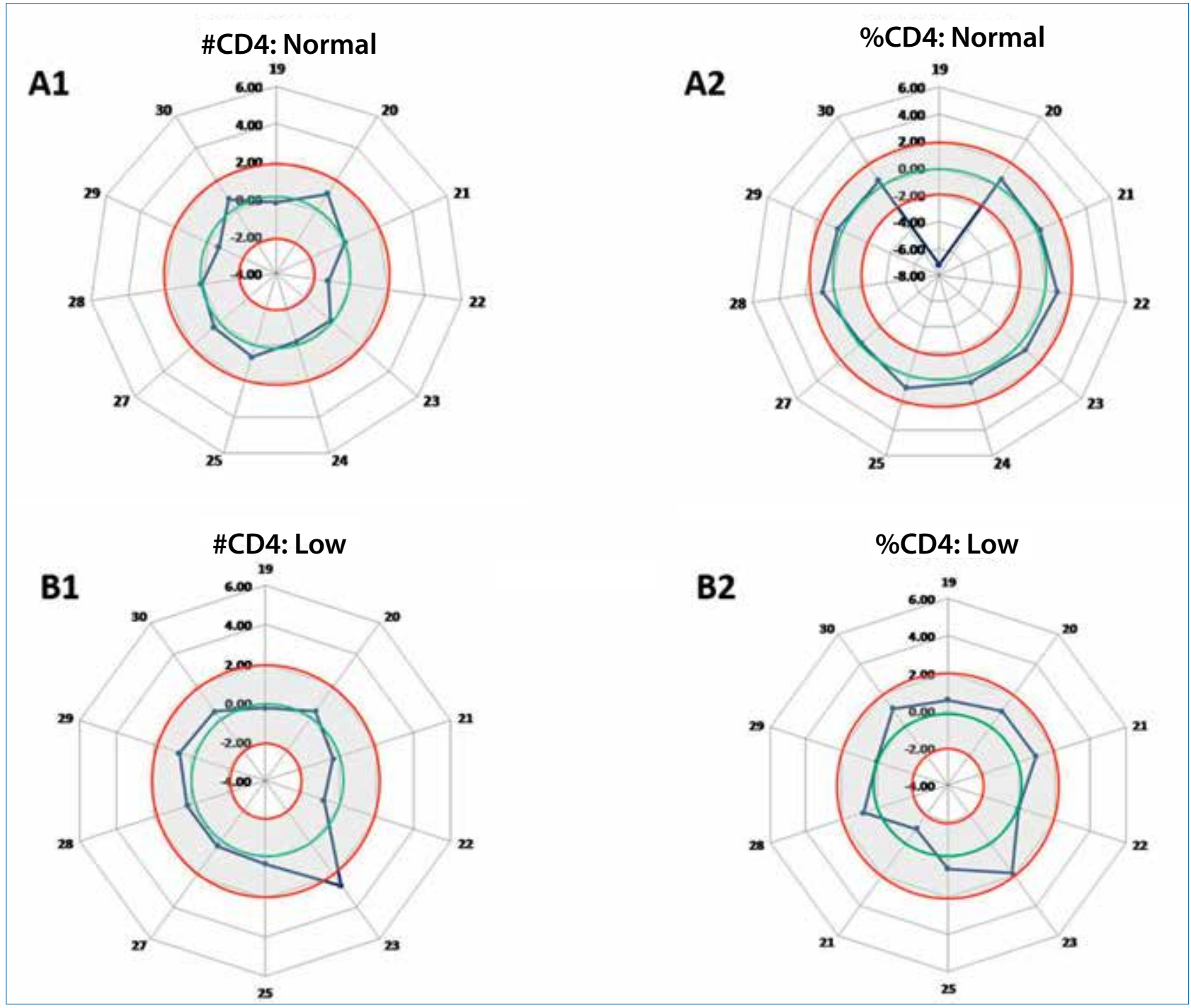

Fig. 4. Performance on EQA after tier 3 CD4 service implementation at De Aar. Radial performance plots show laboratory performance on trials 19 through 30. A1 and A2 represent data from the normal CD4 count proficiency material while B1 and B2 depict results from low CD4 count EQA material. The acceptable range of $-2-+2$ SDI is shaded with a solid line indicating the ideal of zero. An initial outlier on trial 19 was shown to be a transcription error. Another outlier was noted for trial 23 (both parameters). Intervention and corrective action ensured that future performance returned to expected. 
The third service delivery of providing a local small community laboratory was provided for in the ITSDM. This study confirms that the establishment of an ITSDM tier 3 laboratory in a remote, under-serviced area of SA with historically poor TAT can lead to a dramatic improvement of local service delivery. There was a notable reduction in the pre-analytical TAT after CD4 testing was started in the De Aar laboratory, from 18 hours when samples were referred in centrally for testing to the Kimberley laboratory, down to a mean of 1.82 hours post implementation. Although anecdotal, the responses from local clinics were favourable; a moderate increase in the number of CD4 tests requested post implementation (see Fig. 3) attested to this. Despite an initial hiccup of increased analytical/ IN-LAB TAT attributable to learning curve, IN-LAB TAT fell to expected levels as staff became better acquainted with NHLS standardised operating procedures. This is an encouraging aspect, and together with the satisfactory EQA review of the performance of the site, suggests that any small laboratory, with few staff members, is likely to cope well with an add-on tier 3 service. Although some CD4 testing was still referred to the larger metropolitan tier 4 lab at Kimberley after implementation, because referring clinic sites were in closer proximity to this centre (see Fig. 3), a moderately increased local workload (Fig. 3) was noted post implementation at De Aar. This is also an important outcome. If smaller laboratories are capable of an additional modest workload, without major implementation, overhead and staffing costs, this could potentially save the country millions of rands while extending the footprint of laboratory services. ${ }^{[1,8]}$ Previous cost modelling work in Pixley ka Seme district ${ }^{[8]}$ reveals that establishing this small tier 3 site may cost marginally more per CD4 test than a CD4 tested in a centralised larger laboratory (ZAR28.62/USD2.06 at exchange rate of ZAR13.90 per USD on 20 November 2015). This is, however, substantially cheaper than implementing widespread use of POC technology (44 tier 1 facilities) across the district's 44 clinic sites, where it has been estimated that widespread $\mathrm{CD} 4$ provided at the POC could cost Pixley ka Seme in excess of ZAR4.5 million/USD325 700 (at ZAR449.25/USD32.32 per test) as opposed to an estimated ZAR1.03 million/USD74 000 needed to provide a tier 3 laboratory-based service offering the same number of tests performed, but at the De Aar facility. The additional benefit, as a trade-off for the small incremental cost, is that there are less local logistics costs incurred, as testing is performed on-site, coupled with better sample integrity and significantly improved local TAT - all of which has positive implications for better patient management.

\section{Conclusion}

The extended decentralised tier 3/community laboratory implementation at the De Aar laboratory in Pixley ka Seme offers a compromise between cost and accessibility, with a small incremental cost (ZAR28.63/USD2.06) ${ }^{[8]}$ but substantively lower TAT, while maintaining a quality of testing expected from a larger laboratory. Owing to the success of the De Aar pilot site, additional implementation sites, also identified through ITSDM planning, have been proposed to improve and extend services elsewhere in underserviced districts using existing NHLS laboratories, including Aliwal North (Eastern Cape), Lephalale (Limpopo), St Patricks (Eastern Cape) and Vredenburg (Western Cape).

\section{Study limitations}

Owing to the absence of an end-to-end sample tracking system, it was not possible to report TAT for afferent (time from sample collection by the courier at the health facility to registration on the LIS) and efferent (time from result authorisation to result delivery to the health facility) phases of the laboratory value chain. Additionally, for interlaboratory referrals we were unable to identify whether delays were due to courier delays or to pre-analytical receiving office delays.

Acknowledgements. The authors thank Sue Candy and Manfred Tepper at the NHLS CDW for their assistance and support in extracting the test volume and turnaround time data. We would also like to thank the manager and staff of the De Aar laboratory, Janet Scholtz, the regional quality officer, for providing relevant EQA data. The authors thank the NHLS and National Priority Programme for ongoing support. DKG thanks the South African National Research Foundation for Incentive Funding for Rated Researchers.

This work was selected to represent the top $15 \%$ of local innovations, best practices and lessons learned that will help achieve HIV/AIDS UNAIDS proposed 90-90-90 targets in SA, presented at the UNAIDS/ USAIDS/ PEPFAR/HE2RO and National Department of Health collaborative showcase meeting entitled 'Reaching 90-90-90 in South Africa: Innovations and Best Practices', held at the South African HIV/AIDS meeting in Durban during June 2015.

Conflict of interest. DKG declares that the employer, the NHLS, is the sole owner of the patent held for the PanLeucogated CD4 method, currently licensed to Beckman Coulter International and receives royalties. Through open public tender, Beckman Coulter was awarded a service level agreement to provide CD4 testing services in the NHLS.

All LIS sample-related CDW data were used with permission from the NHLS CEO, Dr Sagie Pillay.

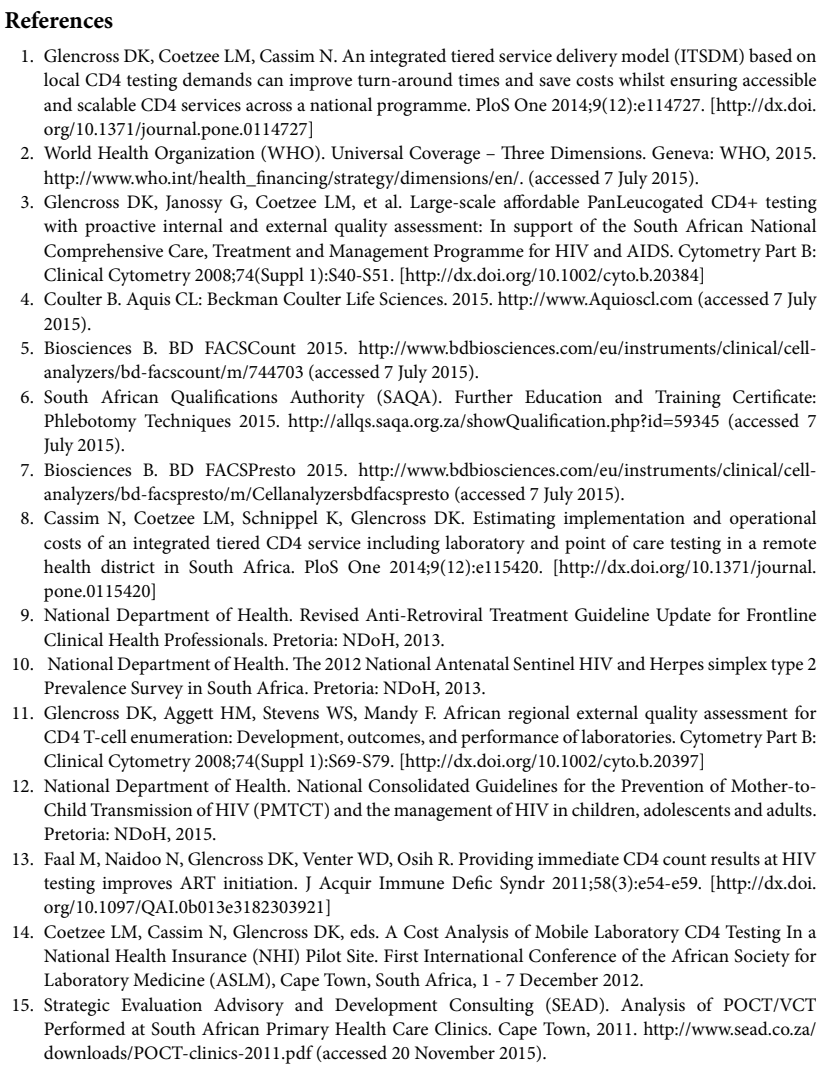

1. Glencross DK, Coetzee LM, Cassim N. An integrated tiered service delivery model (ITSDM) based on local CD4 testing demands can improve turn-around times and save costs whilst ensuring accessible and scalable CD4 services across a national programme. PloS One 2014;9(12):e114727. [http://dx.doi. org/10.1371/journal.pone.0114727]

2. World Health Organization (WHO). Universal Coverage - Three Dimensions. Geneva: WHO, 2015. http://www.who.int/health_financing/strategy/dimensions/en/. (accessed 7 July 2015).

3. Glencross DK, Janossy G, Coetzee LM, et al. Large-scale affordable PanLeucogated CD4+ testing with proactive internal and external quality assessment: In support of the South African National Comprehensive Care, Treatment and Management Programme for HIV and AIDS. Cytometry Part B: Clinical Cytometry 2008;74(Suppl 1):S40-S51. [http://dx.doi.org/10.1002/cyto.b.20384]

4. Coulter B. Aquis CL: Beckman Coulter Life Sciences. 2015. http://www.Aquioscl.com (accessed 7 July 2015).

5. Biosciences B. BD FACSCount 2015. http://www.bdbiosciences.com/eu/instruments/clinical/cell5. Biosciences B. BD FACSCount 2015. http://www.bdbios
analyzers/bd-facscount $/ \mathrm{m} / 744703$ (accessed 7 July 2015).

6. South African Qualifications Authority (SAQA). Further Education and Training Certificate: Phlebotomy Techniques 2015. http://allqs.saqa.org.za/showQualification.php?id=59345 (accessed 7 July 2015

7. Biosciences B. BD FACSPresto 2015. http://www.bdbiosciences.com/eu/instruments/clinical/cellanalyzers/bd-facspresto/m/Cellanalyzersbdfacspresto (accessed 7 July 2015)

8. Cassim N, Coetzee LM, Schnippel K, Glencross DK. Estimating implementation and operational costs of an integrated tiered $\mathrm{CD} 4$ service including laboratory and point of care testing in a remote health district in South Africa. PloS One 2014;9(12):e115420. [http://dx.doi.org/10.1371/journal. pone.0115420]

9. National Department of Health. Revised Anti-Retroviral Treatment Guideline Update for Frontline Clinical Health Professionals. Pretoria: NDoH, 2013.

10. National Department of Health. The 2012 National Antenatal Sentinel HIV and Herpes simplex type 2 Prevalence Survey in South Africa. Pretoria: NDoH, 2013.

11. Glencross DK, Aggett HM, Stevens WS, Mandy F. African regional external quality assessment for CD4 T-cell enumeration: Development, outcomes, and performance of laboratories. Cytometry Part B:
Clat CD4 T-cell enumeration: Development, outcomes, and performance of laboratories. Cyto

12. National Department of Health. National Consolidated Guidelines for the Prevention of Mother-toChild Transmission of HIV (PMTCT) and the management of HIV in children, adolescents and adults. Pretoria: NDoH, 2015

13. Faal M, Naidoo N, Glencross DK, Venter WD, Osih R. Providing immediate CD4 count results at HIV testing improves ART initiation. J Acquir Immune Defic Syndr 2011;58(3):e54-e59. [http://dx.doi. org/10.1097/QAI.0b013e3182303921]

14. Coetzee LM, Cassim N, Glencross DK, eds. A Cost Analysis of Mobile Laboratory CD4 Testing In a National Health Insurance (NHI) Pilot Site. First International Conference of the African Society for Laboratory Medicine (ASLM), Cape Town, South Africa, 1 - 7 December 2012.

15. Strategic Evaluation Advisory and Development Consulting (SEAD). Analysis of POCT/VCT Performed at South African Primary Health Care Clinics. Cape Town, 2011. http://www.sead.co.za/ downloads/POCT-clinics-2011.pdf (accessed 20 November 2015).

Accepted 2 November 2015. 